\title{
Effect of linear polarizers on the behavior of partially coherent and partially polarized highly focused fields
}

\author{
Rosario Martínez-Herrero ${ }^{1}$, David Maluenda ${ }^{2}$, Ignasi Juvells ${ }^{3}$, and Artur Carnicer ${ }^{3, *}$ \\ ${ }^{1}$ Universidad Complutense de Madrid, Facultad de Ciencias Físicas, Departamento de Óptica, Ciudad Universitaria, 28040 Madrid, Spain \\ ${ }^{2}$ Consejo Superior de Investigaciones Científicas (CSIC), Centro Nacional de Biotecnología, Biocomputing Unit, Darwin 3,28049 Madrid, Spain. \\ ${ }^{3}$ Universitat de Barcelona (UB), Facultat de Física, Departament de Física Aplicada, Martí i Franquès 1, 08028 Barcelona, Spain \\ ${ }^{*}$ Corresponding author: artur.carnicer@ub.edu
}

In this letter, we describe the behavior of partially coherent, partially polarized focused vector beams after passing a linear polarizer placed at the focal plane of a high numerical aperture microscope lens. In particular, we develop a mathematical framework for such beams that helps to understand the performance of polarizers when interact with non-paraxial beams. The features of the focused field after the polarizer are numerically evaluated for some illustrative examples. () 2018 Optical Society of America

OCIS codes: (260.2110) Electromagnetic optics; (260.5430) Polarization; (030.1640) Coherence.

\section{http://dx.doi.org/10.1364/ao.XX.XXXXXX}

Generally speaking, when a beam passes through a linear polarizer, the electric field is projected in the direction of the polarizer axis. While this assumption is valid for collimated beams, it becomes inconsistent for tightly focused beams because the electric field may display a non-negligible longitudinal component [1-11]. In a recent paper we showed that an ideal linear polarizer placed at the focal plane of a high numerical aperture (NA) optical system cannot be considered as a simple projector device [12]. Very recently, other authors have developed another model for focused fields interacting with linear polarizers [13]; in both cases, the results are in good agreement. Due to the wide interest that partially coherent partially polarized beams attracts [14-16], the present paper broadens our previous study to more general fields.

In this letter we describe how a partially coherent and partially polarized beam is modified after passing an ideal polarizer placed at the focal plane of a high NA microscope lens. This paper is organized as follows: first, we mathematically describe the behavior of these beams. Then, equations are numerically evaluated for some illustrative examples: we take into account both non-polarized beams and fully polarized Gauss-Schell fields; in particular, azimuthally and radially polarized cases are considered. Finally we present our conclusions.

According to the theory on vector field propagation [17], the electric field at the focal region $\mathbf{E}$ is described by

$$
\mathbf{E}(\mathbf{r})=A \int_{0}^{\theta_{M} 2 \pi} \int_{0} \sqrt{\cos \theta} \mathbf{E}_{0}(\theta, \varphi) \exp (-i k \mathbf{r} \cdot \mathbf{s}) \sin \theta d \theta d \varphi
$$

where $\theta$ and $\varphi$ are the coordinates at the Gaussian sphere of reference, $\mathbf{E}_{0}(\theta, \varphi)$ is the vector angular spectrum, $\mathbf{r}=(r, \phi, z)$ are the coordinates at the focal area, $A$ is a constant, $\theta_{M}$ is the semi-aperture angle, $k$ is the wave-number, and $\mathbf{s}=$ $(\sin \theta \cos \varphi, \sin \theta \sin \varphi, \cos \theta)$ is the wave-front vector. The angular spectrum $\mathbf{E}_{0}(\theta, \varphi)$ is described

$$
\mathbf{E}_{0}(\theta, \varphi)=f_{1}(\theta, \varphi) \mathbf{e}_{1}(\varphi)+f_{2}(\theta, \varphi) \mathbf{e}_{2}(\theta, \varphi)
$$

where $f_{1}(\theta, \varphi)=\mathbf{E}_{s} \cdot \mathbf{e}_{1}$ and $f_{2}(\theta, \varphi)=\mathbf{E}_{s} \cdot \mathbf{e}_{2}^{\mathrm{i}}$ are, respectively, the azimuthal and radial polarization components of the incident beam; $\mathbf{E}_{s}$ is the electric field of the incident beam and vectors $\mathbf{e}_{1}$, $\mathbf{e}_{2}$ and $\mathbf{e}_{2}^{\mathrm{i}}$ are described by

$$
\begin{aligned}
& \mathbf{e}_{1}=(-\sin \varphi, \cos \varphi, 0) \\
& \mathbf{e}_{2}=(\cos \theta \cos \varphi, \cos \theta \sin \varphi,-\sin \theta) \\
& \mathbf{e}_{2}^{\mathrm{i}}=(\cos \varphi, \sin \varphi, 0)
\end{aligned}
$$

(see Fig. 1 for details).

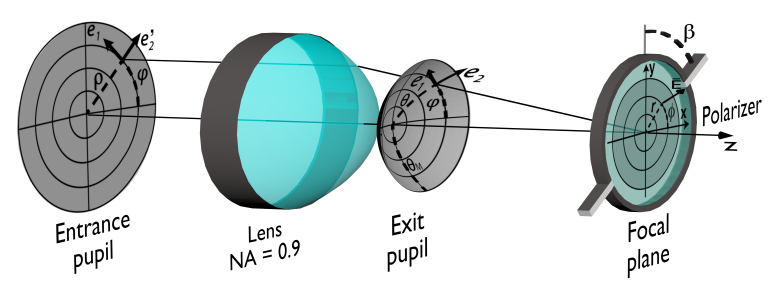

Fig. 1. Coordinate system.

Let us now consider an O-type ideal polarizer with the optical axis ( $c$-axis) described by $(\cos \beta, \sin \beta, 0)$. The electric field after the polarizer is described as $[12,18]$ :

$$
\begin{aligned}
\mathbf{E}(\mathbf{r})=A \int_{0}^{\theta_{M} 2 \pi} \int_{0} \sqrt{\cos \theta}\left(\mathbf{E}_{0}(\theta, \varphi)\right. & \cdot \mathbf{q}(\theta, \varphi, \beta)) \mathbf{p}(\theta, \varphi, \beta) \\
& \times \exp (-i k \mathbf{r} \cdot \mathbf{s}) \sin \theta d \theta d \varphi
\end{aligned}
$$


where vectors $\mathbf{q}(\theta, \varphi, \beta)$, and $\mathbf{p}(\theta, \varphi, \beta)$ are given by

$$
\begin{gathered}
\mathbf{q}(\theta, \varphi, \beta)=t_{s}(\theta) \frac{\cos \theta_{0} \cos (\varphi-\beta)}{\sqrt{1-\sin ^{2} \theta_{0} \cos ^{2}(\varphi-\beta)}} \mathbf{e}_{1}- \\
t_{p}(\theta) \frac{\sin (\varphi-\beta)}{\sqrt{1-\sin ^{2} \theta_{0} \cos ^{2}(\varphi-\beta)}} \mathbf{e}_{2} \\
\mathbf{p}(\theta, \varphi, \beta)=t_{s}^{\prime}(\theta) \frac{\cos \theta_{0} \cos (\varphi-\beta)}{\sqrt{1-\sin ^{2} \theta_{0} \cos ^{2}(\varphi-\beta)}} \mathbf{e}_{1}+ \\
t_{p}^{\prime}(\theta) \frac{\sin ^{2}(\varphi-\beta)}{\sqrt{1-\sin ^{2} \theta_{0} \cos ^{2}(\varphi-\beta)}} \mathbf{e}_{2}
\end{gathered}
$$

and $t_{s}(\theta)\left[t_{s}^{\prime}(\theta)\right]$ and $t_{p}(\theta)\left[t_{p}^{\prime}(\theta)\right]$ are the Fresnel transmission coefficients related to the first [second] surface of the polarizer:

$$
\begin{array}{ll}
t_{s}(\theta)=\frac{2 \cos \theta}{\cos \theta+n_{o} \cos \theta_{0}} & t_{p}(\theta)=\frac{2 \cos \theta}{\cos \theta_{0}+n_{o} \cos \theta} \\
t_{s}^{\prime}(\theta)=\frac{2 n_{o} \cos \theta_{0}}{\cos \theta+n_{o} \cos \theta_{0}} & t_{p}^{\prime}(\theta)=\frac{2 n_{o} \cos \theta_{0}}{\cos \theta_{0}+n_{o} \cos \theta} .
\end{array}
$$

Note that $\theta_{0}$ is the refraction angle, i.e. $\sin \theta=n_{0} \sin \theta_{0}$, and $n_{0}$ is the ordinary refractive index of the polarizer.

The $2 \times 2$ cross-spectral density matrix $(\mathrm{CDM}) \hat{W}_{i}$ for a paraxial partially coherent, partially polarized incident beam described by stochastic processes $f_{1}(\theta, \varphi)$ and $f_{2}(\theta, \varphi)$ is

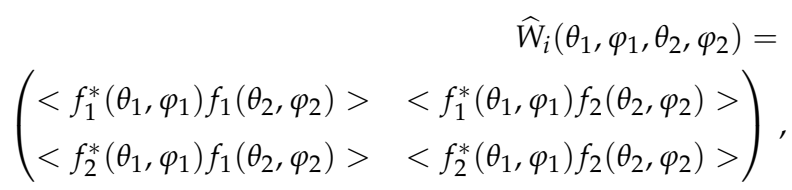

where $\hat{W}_{i}$ is written using the base $\mathbf{e}_{1}-\mathbf{e}_{2}$. This beam passes through an ideal linear polarizer placed at the focal plane of a high NA objective lens. According to Eq. (4) and after some algebra, the propagated CDM $\widehat{W}_{p}\left(\mathbf{r}_{1}, \mathbf{r}_{2}\right)$ reads

$$
\begin{aligned}
& \widehat{W}_{p}\left(\mathbf{r}_{1}, \mathbf{r}_{2}\right)=|A|^{2} \int_{0}^{\theta_{M}} \int_{0}^{\theta_{M}} \int_{0}^{2 \pi 2 \pi} \int_{0}^{2 \pi} \widehat{W}_{o}\left(\theta_{1}, \varphi_{1}, \theta_{2}, \varphi_{2}\right) \times \\
& \times \exp \left(i k\left(\mathbf{r}_{1} \mathbf{s}_{1}-\mathbf{r}_{2} \mathbf{s}_{2}\right)\right) \sin \theta_{1} \sin \theta_{2} d \theta_{1} d \theta_{2} d \varphi_{1} d \varphi_{2}
\end{aligned}
$$

where

$$
\begin{aligned}
\widehat{W}_{o}\left(\theta_{1}, \varphi_{1}, \theta_{2}, \varphi_{2}\right)=\sqrt{\cos \theta_{1}} & \sqrt{\cos \theta_{2}} \Gamma\left(\theta_{1}, \varphi_{1}, \theta_{2}, \varphi_{2}\right) \\
& \times \mathbf{p}^{\dagger}\left(\theta_{1}, \varphi_{1}, \beta\right) \mathbf{p}\left(\theta_{2}, \varphi_{2}, \beta\right) .
\end{aligned}
$$

and $\Gamma\left(\theta_{1}, \varphi_{1}, \theta_{2}, \varphi_{2}\right)$ is a scalar function given by

$$
\Gamma\left(\theta_{1}, \varphi_{1}, \theta_{2}, \varphi_{2}\right)=\mathbf{q}\left(\theta_{1}, \varphi_{1}\right) \widehat{W}_{i}\left(\theta_{1}, \varphi_{1}, \theta_{2}, \varphi_{2}\right) \mathbf{q}^{\dagger}\left(\theta_{2}, \varphi_{2}\right) .
$$

Using the well-known coherent-mode expansion $[19,20], \widehat{W}_{i}$ can be written as a incoherent superposition of fully coherent-fully polarized fields described by vectors $\mathbf{F}_{n}$

$$
\widehat{W}_{i}\left(\theta_{1}, \varphi_{1}, \theta_{2}, \varphi_{2}\right)=\sum_{n} \lambda_{n}^{2} \mathbf{F}_{n}^{\dagger}\left(\theta_{1}, \varphi_{1}\right) \mathbf{F}_{n}\left(\theta_{2}, \varphi_{2}\right) \text {. }
$$

Then, combining Eqs. (8) to (11), the CDM after the polarizer reads

$$
\begin{aligned}
\widehat{W}_{P}\left(\mathbf{r}_{\mathbf{1}}, \mathbf{r}_{\mathbf{2}}\right) & =\sum_{n} \lambda_{n}^{2} \mathbf{H}_{n}^{\dagger}\left(\mathbf{r}_{\mathbf{1}}\right) \mathbf{H}_{n}\left(\mathbf{r}_{\mathbf{2}}\right), \text { with } \\
\mathbf{H}_{n}(\mathbf{r}) & =\int_{0}^{\theta_{M}} \int_{0}^{2 \pi} \sqrt{\cos \theta}\left(\mathbf{F}_{n}(\theta, \varphi) \cdot \mathbf{q}(\theta, \varphi)\right) \mathbf{p}(\theta, \varphi) \\
& \exp (-i k \mathbf{r s}) \sin \theta d \theta d \varphi
\end{aligned}
$$

Equations (8) and (12) are one of the main results of this paper. They provide the relationship between the CDMs of the incident beam and the beam after the polarizer within the framework of partially coherent and partially polarized highly focused beams. According to these equations, it can be concluded that the focused field after the polarizer is partially coherent and displays a non-uniform 3D polarization. This statement is based on the fact that the focused field is not uniformly totally polarized, even when the incident field is totally coherent and totally polarized, as we theoretically and experimentally reported in reference [12]. In fact, the characteristics of this field after the polarizer depend on the spatial structure, coherence and polarization of the incident beam, as we show in the remainder of the paper.

Now, we focus on analyzing the polarization characteristics of the resulting field after the polarizer. We use the 3D degree of polarization described by [21]

$$
P_{3 D}^{2}=\frac{3}{2}\left(\frac{\operatorname{Tr}\left[\widehat{W}_{p}(\mathbf{r}, \mathbf{r}) \widehat{W}_{p}(\mathbf{r}, \mathbf{r})\right]}{\left[\operatorname{Tr}\left[\widehat{W}_{p}(\mathbf{r}, \mathbf{r})\right]\right]^{2}}-\frac{1}{3}\right),
$$

where operator $\operatorname{Tr}[]$ stands for the matrix trace. Using the coherent-mode expansion [Eq. (12a)], the 3D degree of polarization can be written as

$$
P_{3 D}^{2}=1-\frac{3 \sum_{n \neq m} \lambda_{n}^{2} \lambda_{m}^{2}\left|\mathbf{H}_{n}(\mathbf{r})\right|^{2}\left|\mathbf{H}_{m}(\mathbf{r})\right|^{2} \sin ^{2} \alpha_{n m}(\mathbf{r})}{2\left(\sum_{n} \lambda_{n}^{2}\left|\mathbf{H}_{n}(\mathbf{r})\right|^{2}\right)^{2}}
$$

where $\alpha_{n m}(\mathbf{r})$ is the angle between vectors $\mathbf{H}_{n}(\mathbf{r})$ and $\mathbf{H}_{m}(\mathbf{r})$, i.e.:

$$
\sin ^{2} \alpha_{n m}(\mathbf{r})=1-\frac{\left|\mathbf{H}_{n}(\mathbf{r}) \mathbf{H}_{m}^{+}(\mathbf{r})\right|^{2}}{\left|\mathbf{H}_{n}(\mathbf{r})\right|^{2}\left|\mathbf{H}_{m}(\mathbf{r})\right|^{2}} .
$$

Interestingly, for small angles of incidence and if the effect of the Fresnel coefficients [Eq. (6)] is neglected, vectors $\mathbf{H}_{n}(\mathbf{r})$ are parallel to the polarizer axis direction (i.e. $\alpha_{n m}=0$ ) and therefore $P_{3 \mathrm{D}}=1$. In this case, we recover the conventional projector character of the polarizer. Moreover, if the incident beam is totally coherent and fully polarized, then we trivially obtain $P_{3 D}=1$, as described in [12].

Since the set of angles $\alpha_{n m}(\mathbf{r})$ depend on the physical characteristics of the incident field, it can be difficult to a priori determine the behavior of $P_{3 \mathrm{D}}$ in a general case. In order to provide more insight, from now on we analyze beams with CDMs expressed as

$$
\hat{W}_{i}\left(\theta_{1}, \varphi_{1}, \theta_{2}, \varphi_{2}\right)=\Gamma_{i}\left(\theta_{1}, \varphi_{1}, \theta_{2}, \varphi_{2}\right) \hat{M}
$$

with $\hat{M}=\hat{U}^{\dagger} \hat{D} \hat{U}$. $\hat{U}$ is a constant unitary matrix and $\hat{D}$ is a diagonal matrix

$$
\hat{D}=\left(\begin{array}{ll}
a & 0 \\
0 & b
\end{array}\right) a, b \geq 0 .
$$

The coherence properties of this class of fields (sometimes referred as pseudo-scalar beams [22]) are somewhat decoupled 
from their polarization properties [23]. In fact, the conventional 2D degree of polarization of the fields described by Eq. (16) is independent of $\mathbf{r}$ and fully described by matrix $\hat{D}$. Moreover, the maximum Young's experiment visibility that can be achieved with these beams by means of local unitary transformations is given by [24, 25]:

$$
\left|\mu_{W}\right|_{\max }=\frac{\left|\Gamma_{i}\left(\theta_{1}, \phi_{1}, \theta_{2}, \phi_{2}\right)\right|}{\sqrt{\Gamma_{i}\left(\theta_{1}, \phi_{1}, \theta_{1}, \phi_{1}\right) \Gamma_{i}\left(\theta_{2}, \phi_{2}, \theta_{2}, \phi_{2}\right)}} .
$$

According to the properties of function $\Gamma_{i}()$, we refer to coherent incident beams when $\Gamma_{i}()$ is factorizable, and partially coherent in other cases. In particular, for beams with $\Gamma_{i}\left(\theta_{1}, \varphi_{1}, \theta_{2}, \varphi_{2}\right)=$ $g^{*}\left(\theta_{1}, \varphi_{1}\right) g\left(\theta_{2}, \varphi_{2}\right)$, the $3 \mathrm{D}$ degree of polarization reads

$$
P_{3 D}^{2}=1-\frac{3 a b\left|\mathbf{H}_{1}(\mathbf{r})\right|^{2}\left|\mathbf{H}_{2}(\mathbf{r})\right|^{2} \sin ^{2} \alpha_{12}(\mathbf{r})}{\left(a\left|\mathbf{H}_{1}(\mathbf{r})\right|^{2}+b\left|\mathbf{H}_{2}(\mathbf{r})\right|^{2}\right)^{2}}
$$

where

$$
\begin{array}{r}
\mathbf{H}_{j}(\mathbf{r})=A \int_{0}^{\theta_{M}} \int_{0}^{2 \pi} \sqrt{\cos \theta}\left(\mathbf{q} U^{\dagger}\right)_{j}^{*} g(\theta, \varphi) \mathbf{p}(\theta, \varphi) \times \\
\times \exp (-i k \mathbf{r s}) \sin \theta d \theta d \varphi
\end{array}
$$

with $j=1$, 2. According to Eq. (19), $P_{3 D}$ depends on angle $\alpha_{12}(\mathbf{r})$ between $\mathbf{H}_{1}(\mathbf{r})$ and $\mathbf{H}_{2}(\mathbf{r})$, the modulus of these vectors and values $a$ and $b$ from matrix $D$. Interestingly, in the present case, it can be proven that $P_{3 D}(\mathbf{r})>0.5 \forall \mathbf{r}$. Therefore, for a coherent and uniform partially polarized incident beam and regardless its degree of polarization, the field after the polarizer becomes nonuniformly partially polarized with $\min \left\{P_{3 D}\right\}=0.5$. This means that the field after the polarizer is not a genuine $3 \mathrm{D}$ optical field [26].

In order to illustrate the behavior of these class of beams, Figure 2 displays the irradiance of the field components, the total intensity, angle $\alpha_{12}$ and $P_{3 D}$ for a doughnut-shaped beam with topological charge equal to 1 with the following profile:

$$
g(\theta, \varphi)=\exp \left(-\frac{\sin ^{2} \theta}{f_{o}^{2} \sin ^{2} \theta_{M}}\right) \sin \theta e^{i \varphi}
$$

The calculation has been carried out using a focusing lens $\mathrm{NA}=0.9\left(\sin \theta_{M}=64^{\circ}\right)$ and a filling factor $f_{0}=1$. The filling factor is related with the focal length $f$ of the focusing system and the beam waist $w_{0}$ by means of $f_{o}=\frac{w_{0}}{f \sin \theta_{M}}$. In the studied case matrix $U$ is the identity and thus, $\hat{M}_{i}=\hat{D}$, and $a=b=1$; polarization angle is set to $\beta=0$. Note that the degree of polarization $P_{3 D}$ displays the same shape as angle $\alpha_{12}$. As expected, $P_{3 D}>0.5$.

Now if the field is partially coherent and totally polarized $(a=1, b=0)$ or $(a=0, b=1)$ i.e.

$$
\Gamma_{i}\left(\theta_{1}, \varphi_{1}, \theta_{2}, \varphi_{2}\right)=\sum_{n} \lambda_{n}^{2} g_{n}\left(\theta_{1}, \varphi_{1}\right) g_{n}\left(\theta_{2}, \varphi_{2}\right),
$$

the $3 \mathrm{D}$ degree of polarization $P_{3 D}$ reads

$$
P_{3 D j}^{2}=1-\frac{3 \sum_{n \neq m} \lambda_{n}^{2} \lambda_{m}^{2}\left|\mathbf{H}_{n j}(\mathbf{r})\right|^{2}\left|\mathbf{H}_{m j}(\mathbf{r})\right|^{2} \sin ^{2} \alpha_{n m j}(\mathbf{r})}{2\left(\sum_{n} \lambda_{n}^{2}\left|\mathbf{H}_{n j}(\mathbf{r})\right|^{2}\right)^{2}}
$$

with

$$
\begin{array}{r}
\mathbf{H}_{n j}(\mathbf{r})=A \int_{0}^{\theta_{M}} \int_{0}^{2 \pi} \sqrt{\cos \theta}\left(\mathbf{q} U^{\dagger}\right)_{j}^{*} g_{n}(\theta, \varphi) \mathbf{p}(\theta, \varphi) \times \\
\times \exp (-i k \mathbf{r s}) \sin \theta d \theta d \varphi,
\end{array}
$$

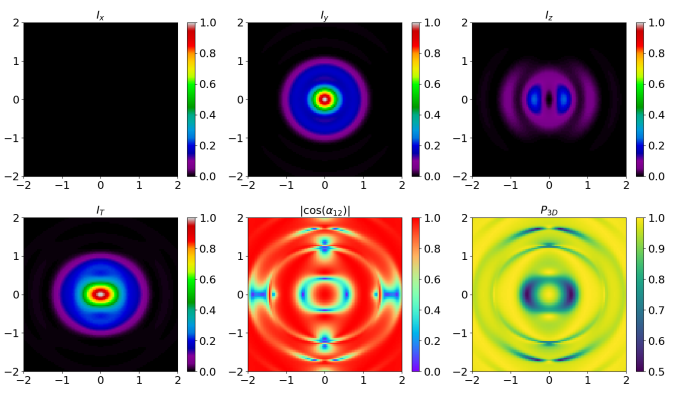

Fig. 2. Non-polarized coherent beam $a=b=1, \mathrm{NA}=0.9$, $f_{0}=1$ and $\beta=0$. The minimum value of $P_{3 D}$ is 0.52 . These distributions are calculated at the plane $x-y$ (see Fig. 1). Axes values are shown in $\lambda$ units. Intensities are normalized to the maximum of the total irradiance.

and $j=1,2 ; j=1(2)$ stands for the azimuthal(radial) polarization. Although Eqs. (23) and (14) are formally equivalent, they provide different physical information. Here the fundamental role is played by the angles that form fields with identical initial polarization but with different spatial distributions. In other words, the response of the polarizer to different spatial profiles is what in this case produces a non-uniform partially polarized field after the polarizer.

To illustrate the behavior of a totally polarized partially coherent focused beam passing through a polarizer in the focal plane, we use a Gauss-Schell source [27] with topological charge equal to 1 [28]. The CDM of this field reads

$$
\begin{gathered}
\Gamma_{i}\left(\theta_{1}, \varphi_{1}, \theta_{2}, \varphi_{2}\right) \propto \frac{\sin \theta_{1} \sin \theta_{2}}{f_{0}^{2} \sin ^{2} \theta_{M}} \exp \left(-\frac{\sin ^{2} \theta_{1}+\sin ^{2} \theta_{2}}{f_{o}^{2} \sin ^{2} \theta_{M}}\right) \times \\
\times \exp \left(-\frac{\sin ^{2} \theta_{1}+\sin ^{2} \theta_{2}-2 \sin \theta_{1} \sin \theta_{2} \cos \left(\varphi_{1}-\varphi_{2}\right)}{L_{c}^{2} / f^{2}}\right) \times \\
\times \exp \left(i\left(\varphi_{2}-\varphi_{1}\right)\right) .
\end{gathered}
$$

$L_{c} / f$ is the transverse longitude of coherence of the source divided by the focal length; in particular, $L_{c} / f \rightarrow 0$ and $L_{c} / f \rightarrow \infty$ describe totally incoherent and totally coherent sources respectively.

For illustrative purposes, we present several calculations using Eqs. (8) and (13). For each considered case, we show (i) the diagonal components of $\hat{W}_{p}(\mathbf{r}, \mathbf{r})$ that explain the intensity of the beam in the three Cartesian directions, i.e. $I_{x}, I_{y}$ and $I_{z}$, (ii) the transversal part $I_{t}=I_{x}+I_{y}$ and the total intensity $I_{T}=I_{x}+I_{y}+I_{Z}$ and (iii) the square of the 3-D Polarization degree $P_{3 D}$. Figure 3 displays the results for partially coherent beams with a very small degree of coherence $\left(L_{c} / f=0.3\right)$ focused with an objective lens NA=0.9 $\left(\theta_{M} \approx 64^{\circ}\right)$ and $f_{0}=1$ : in Fig. 3a the incident beam is radially polarized. In this case, $I_{x} \approx 0$ (the polarizer is set in the $y$-direction $(\beta=0)$. Interestingly, $P_{3 D}$ is nearly constant with $P_{3 D} \approx 0.5$; this means that the radially polarized beam is greatly depolarized. Fig. $3 b$ shows the results for an azimuthally polarized beam. In this case $I_{x} \approx 0, I_{z}$ displays low values and $P_{3 D} \approx 0.6$. Again the beam becomes depolarized after passing through the polarizer. In both cases, these fields do not behave as genuine 3D optical fields. As expected, the maximum value of $I_{z}$ for the radially polarized case is higher that the corresponding one in the azimuthal case. Fig. 4 provides information of the behavior of a highly focused partially coherent beam with $L_{c} / f=3$. In 
the two cases considered (radial and azimuthal polarization), $P_{3 D}$ displays a complicated spatial pattern that depends on the local values of the intensity components and the angles between involved vectors $\mathbf{H}_{n}$. Fig. 4 has been calculated in the same conditions as Fig. 3. From the analysis of Figs. 3 and 4 it can be concluded that as coherence increases, that is the number of significant terms in Eq. (23) decreases, the $P_{3 D}$ landscape is less homogeneous. However, condition $P_{3 D}>0.5$ always hold.
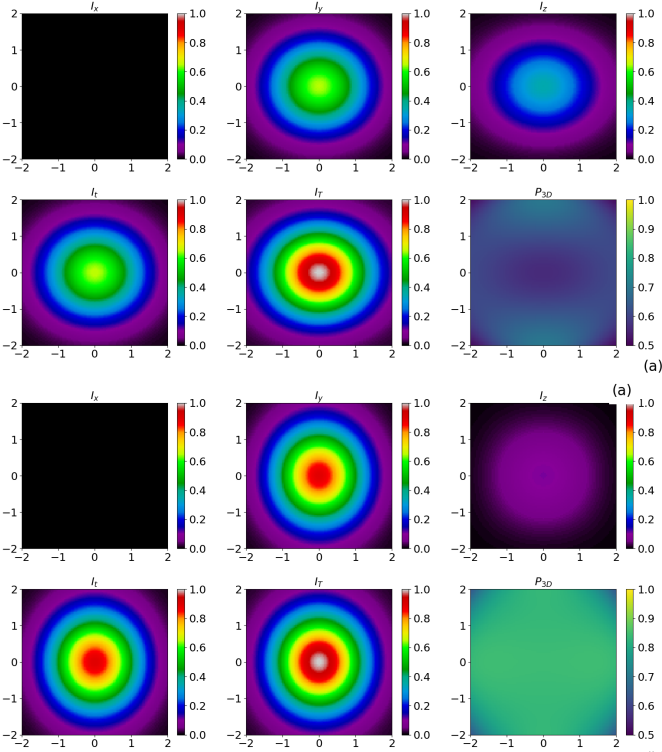

Fig. 3. Almost incoherent beam $\left(L_{c} / f=0.3\right), \mathrm{NA}=0.9, f_{0}=1$, $\beta=0$ : (a) radially polarized $(a=0, b=1), \min \left(P_{3 D}\right)=0.51$; (b) azimuthally polarized $(a=1, b=0), \min \left(P_{3 D}\right)=0.64$. These distributions are calculated at the plane $x-y$ (see Fig. 1). Axes values are shown in $\lambda$ units. Intensities are normalized to the maximum of the total irradiance.

In summary, we derived an analytical expression for the CDM of the electromagnetic field after passing a linear polarizer placed at the focal plane of a high NA lens when the incoming beam is partially coherent and partially polarized. In general, the focused field after the polarizer displays a three dimensional structure and is non uniformly partially polarized. As expected, the polarization properties of this field depend on the spatial distribution and the coherence and polarization characteristics of the incident field. To illustrate the features of the focused field after the polarizer, we took into account a pseudo scalar incident beam. In the cases considered, we found that the $P_{3 D}$ displays an inhomogeneous spatial distribution. Nevertheless, $P_{3 D}>0.5$ that means the field cannot be considered a genuine 3D optical field. On the other hand, when the incident beam is partially coherent and totally polarized, the focused beam after the polarized is depolarized. This effect is more noticeable for incident radially polarized beams.

Funding: $\mathrm{RMH}$ and $\mathrm{AC}$ acknowledges support from MINECO project number FIS2016-75147-C3-1-P.

\section{REFERENCES}

1. Y. Fainman and J. Shamir, App. Opt. 23, 3188 (1984).

2. A. Aiello, C. Marquardt, and G. Leuchs, Opt. Let. 34, 3160 (2009).

3. J. Korger, T. Kolb, P. Banzer, A. Aiello, C. Wittmann, C. Marquardt, and G. Leuchs, Opt. Express 21, 27032 (2013).

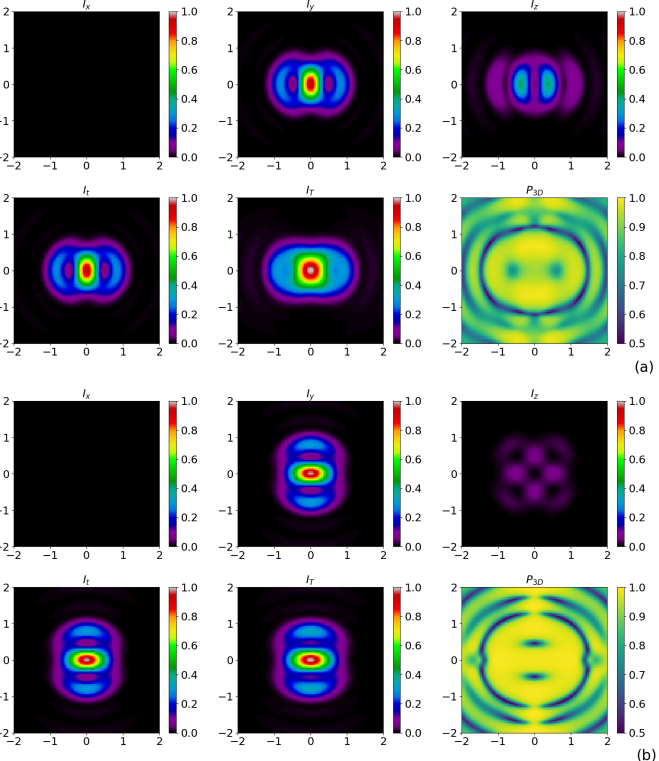

Fig. 4. Partially coherent beam $\left(L_{c} / f=3\right), \mathrm{NA}=0.9, f_{0}=1$, $\beta=0$ : (a) radially polarized $(a=0, b=1), \min \left(P_{3 D}\right)=0.5$; (b) azimuthally polarized $(a=1, b=0), \min \left(P_{3 D}\right)=0.5$. These distributions are calculated at the plane $x-y$ (see Fig. 1). Axes values are shown in $\lambda$ units. Intensities are normalized to the maximum of the total irradiance.

4. P. Yeh, J. Opt. Soc. Am. 69, 742 (1979).

5. D. W. Berreman, J. Opt. Soc. Am. 62, 502 (1972).

6. P. Yeh, Surf. Sci. 96, 41 (1980).

7. P. Yeh, J. Opt. Soc. Am. 72, 507 (1982).

8. C. Gu and P. Yeh, J. Opt. Soc. Am. A 10, 966 (1993).

9. P. Yeh, Optical waves in layered media, vol. 95 (Wiley New York, 1988).

10. H. L. Ong, Appl. Phys. Lett. 59, 155 (1991).

11. H. L. Ong, Jpn. J. Appl. Phys 30, L1028 (1991).

12. R. Martínez-Herrero, D. Maluenda, I. Juvells, and A. Carnicer, Sci. Rep. 7, 42122 (2017).

13. S. Zhang, H. Partanen, C. Hellmann, and F. Wyrowski, Opt. Express 26, 9840 (2018).

14. G. Gbur and T. Visser, "The structure of partially coherent fields," in "Progress in optics," , vol. 55 (Elsevier, 2010), pp. 285-341.

15. Y. Cai, Y. Chen, J. Yu, X. Liu, and L. Liu, "Generation of partially coherent beams," in "Progress in Optics," , vol. 62 (Elsevier, 2017), pp. 157-223.

16. A. T. Friberg and T. Setälä, J. Opt. Soc. Am. A 33, 2431 (2016).

17. B. Richards and E. Wolf, P. Roy. Soc. London A Mat. 253, 358 (1959).

18. R. Martínez-Herrero, D. Maluenda, I. Juvells, and A. Carnicer, Opt. Lasers Eng. 98, 176 (2017).

19. J. Tervo, T. Setälä, and A. T. Friberg, J. Opt. Soc. Am. A 21, 2205 (2004).

20. R. Martínez-Herrero and P. M. Mejías, Opt. Lett. 34, 2303 (2009).

21. T. Setälä, A. Shevchenko, M. Kaivola, and A. T. Friberg, Phys. Rev. E 66, 016615 (2002).

22. R. Martínez-Herrero, P. M. Mejías, and G. Piquero, Characterization of partially polarized light fields, vol. 147 (Springer Science \& Business Media, 2009).

23. M. Santarsiero, J. Opt. Soc. Am. A 24, 3493 (2007).

24. R. Martínez-Herrero and P. M. Mejías, Opt. Lett. 32, 1471 (2007).

25. R. Martínez-Herrero and P. M. Mejías, Opt. Lett. 32, 1504 (2007).

26. T. Setälä, K. Lindfors, and A. T. Friberg, Opt. Lett. 34, 3394 (2009).

27. L. Mandel and E. Wolf, Optical coherence and quantum optics (Cambridge university press, 1995).

28. X. Liu, F. Wang, L. Liu, C. Zhao, and Y. Cai, J. Opt. Soc. Am. A 32, 2058 (2015) 


\section{REFERENCES}

1. Y. Fainman and J. Shamir, "Polarization of nonplanar wave fronts," App. Opt. 23, 3188-3195 (1984).

2. A. Aiello, C. Marquardt, and G. Leuchs, "Nonparaxial polarizers," Opt. Let. 34, 3160-3162 (2009).

3. J. Korger, T. Kolb, P. Banzer, A. Aiello, C. Wittmann, C. Marquardt, and G. Leuchs, "The polarization properties of a tilted polarizer," Opt. Express 21, 27032-27042 (2013).

4. P. Yeh, "Electromagnetic propagation in birefringent layered media," J. Opt. Soc. Am. 69, 742-756 (1979).

5. D. W. Berreman, "Optics in stratified and anisotropic media: $4 \times 4$-matrix formulation," J. Opt. Soc. Am. 62, 502-510 (1972).

6. P. Yeh, "Optics of anisotropic layered media: a new $4 \times 4$ matrix algebra," Surf. Sci. 96, 41-53 (1980).

7. P. Yeh, "Extended Jones matrix method," J. Opt. Soc. Am. 72, 507-513 (1982).

8. C. Gu and P. Yeh, "Extended Jones matrix method II," J. Opt. Soc. Am. A 10, 966-973 (1993).

9. P. Yeh, Optical waves in layered media, vol. 95 (Wiley New York, 1988).

10. H. L. Ong, "Electro-optics of electrically controlled birefringence liquidcrystal displays by $2 \times 2$ propagation matrix and analytic expression at oblique angle," Appl. Phys. Lett. 59, 155-157 (1991).

11. H. L. Ong, "Electro-optics of a twisted nematic liquid crystal display by $2 \times 2$ propagation matrix at oblique angle," Jpn. J. Appl. Phys 30, L1028 (1991).

12. R. Martínez-Herrero, D. Maluenda, I. Juvells, and A. Carnicer, "Polarisers in the focal domain: Theoretical model and experimental validation," Sci. Rep. 7, 42122 (2017).

13. S. Zhang, H. Partanen, C. Hellmann, and F. Wyrowski, "Non-paraxial idealized polarizer model," Opt. Express 26, 9840-9849 (2018)

14. G. Gbur and T. Visser, "The structure of partially coherent fields," in "Progress in optics,", vol. 55 (Elsevier, 2010), pp. 285-341.

15. Y. Cai, Y. Chen, J. Yu, X. Liu, and L. Liu, "Generation of partially coherent beams," in "Progress in Optics," , vol. 62 (Elsevier, 2017), pp. 157-223.

16. A. T. Friberg and T. Setälä, "Electromagnetic theory of optical coherence," J. Opt. Soc. Am. A 33, 2431-2442 (2016).

17. B. Richards and E. Wolf, "Electromagnetic diffraction in optical systems. ii. structure of the image field in an aplanatic system," P. Roy. Soc. London A Mat. 253, 358-379 (1959).

18. R. Martínez-Herrero, D. Maluenda, I. Juvells, and A. Carnicer, "Effect of linear polarizers on highly focused spirally polarized fields," Opt. Lasers Eng. 98, 176-180 (2017).

19. J. Tervo, T. Setälä, and A. T. Friberg, "Theory of partially coherent electromagnetic fields in the space-frequency domain," J. Opt. Soc. Am. A 21, 2205-2215 (2004).

20. R. Martínez-Herrero and P. M. Mejías, "Elementary-field expansions of genuine cross-spectral density matrices," Opt. Lett. 34, 2303-2305 (2009).

21. T. Setälä, A. Shevchenko, M. Kaivola, and A. T. Friberg, "Degree of polarization for optical near fields," Phys. Rev. E 66, 016615 (2002).

22. R. Martínez-Herrero, P. M. Mejías, and G. Piquero, Characterization of partially polarized light fields, vol. 147 (Springer Science \& Business Media, 2009).

23. M. Santarsiero, "Polarization invariance in a young interferometer," J. Opt. Soc. Am. A 24, 3493-3499 (2007).

24. R. Martínez-Herrero and P. M. Mejías, "Maximum visibility under unitary transformations in two-pinhole interference for electromagnetic fields," Opt. Lett. 32, 1471-1473 (2007).

25. R. Martínez-Herrero and P. M. Mejías, "Relation between degrees of coherence for electromagnetic fields," Opt. Lett. 32, 1504-1506 (2007).

26. T. Setälä, K. Lindfors, and A. T. Friberg, "Degree of polarization in 3d optical fields generated from a partially polarized plane wave," Opt. Lett. 34, 3394-3396 (2009).

27. L. Mandel and E. Wolf, Optical coherence and quantum optics (Cambridge university press, 1995).

28. X. Liu, F. Wang, L. Liu, C. Zhao, and Y. Cai, "Generation and propagation of an electromagnetic gaussian schell-model vortex beam," J. Opt. 